\title{
Desarrollo y validación de un método analítico para la determinación de N-3-metoxibencil-linoleamida en homogenizado de cerebro de ratas Sprague Dawley por Cromatografía líquida de alta resolución
}

Development and validation of an analytical method for the determination of N-3-methoxybenzyllinoleamide in brain homogenate of Sprague Dawley rats by High Performance Liquid Chromatography

Rodrigo Moscoso Palacios ${ }^{\mathrm{a}}$, Maria Aranzamendi Zenteno ${ }^{\mathrm{a}}$, Julitza Paredes Fuentes ${ }^{\mathrm{b}}$, José Carpio Carpio ${ }^{\mathrm{b}}$, José Villanueva Salas ${ }^{\mathrm{b}}$, Karin Vera López ${ }^{\mathrm{a}}$ y Rita Nieto Montesinos ${ }^{\mathrm{a}}$

aLaboratorio de Neurociencias y Tecnología Farmacéutica, Universidad Católica de Santa María, Arequipa, Perú.

bLaboratorio de Biología Molecular, Universidad Católica de Santa María, Arequipa, Perú.

\section{INFORMACIÓN}

\section{Historia del Artículo}

Recepción: 18/09/2019

Revisión: 10/10/2019

Aceptación: 20/11/2019

\section{Palabras Clave}

N-3-metoxibencil-linoleamida, homogenizado de cerebro de rata, HPLC y validación

\section{Key Words}

N-3-methoxybencyl linoleamida, rat brain homogenate, HPLC and validation

\section{DOI}

https://doi.org/10.35286/veritas. v21i1.264

\begin{abstract}
RESUMEN
Un método rápido, sensible y preciso por Cromatografía Líquida de Alta Resolución fue desarrollado y validado para la determinación de los niveles de N-3-metoxibencil-linoleamida en cerebro de ratas Sprague Dawley. Durante la preparación de la muestra se usó acetonitrilo como solvente de extracción y N-bencil-palmitamida como estándar interno. El analito se separó en una columna Chromolith $®$ Performance con elución isocrática de acetonitrilo-agua ultrapura $(80: 20, \mathrm{v} / \mathrm{v})$ a $1.0 \mathrm{~mL} / \mathrm{min}$ y a $40^{\circ} \mathrm{C}$. La detección se realizó utilizando un detector de arreglo de diodos a una longitud de onda de $205 \mathrm{~nm}$. La selectividad del método se confirmó por la ausencia de interferentes endógenos. El método desarrollado se usó para construir curvas de calibración lineales en el rango de concentración de 250 y $10000 \mathrm{ng} / \mathrm{mL}$, (R2 $\geq 0.9900)$. Usando $500 \mu \mathrm{g}$ de homogeneizado de cerebro, el límite inferior de cuantificación validado fue de $250.0 \mathrm{ng} /$ $\mathrm{mL}$. La exactitud y precisión intra e inter-día estuvieron dentro del $15 \%$ de las concentraciones nominales. Asimismo, el analito y su estándar interno demostraron ser estables durante el almacenamiento, la preparación de muestra y el análisis cromatográfico.
\end{abstract}

\begin{abstract}
A rapid, sensitive, precise and accurate High Performance Liquid Chromatography method was developed and validated for the determination of N-3-methoxybenzyl-linoleamide levels in Sprague Dawley rat brain. During sample preparation, acetonitrile was used as extraction solvent and N-benzyl-palmitamide as internal standard. The analyte was separated on a Chromolith ${ }^{\circledR}$ Performance column with isocratic elution of acetonitrile-ultrapure water $(80: 20, \mathrm{v} / \mathrm{v})$ at $1.0 \mathrm{~mL} /$ min and $40^{\circ} \mathrm{C}$. Detection was performed using a diode array detector at a wavelength of $205 \mathrm{~nm}$. The selectivity of the method was confirmed by the absence of interferences from endogenous compounds. The developed method was used to construct linear calibration curves over the concentration range of 250 and $10000 \mathrm{ng} / \mathrm{mL}(\mathrm{R} 2 \geq 0.9900)$. Using $500 \mu \mathrm{g}$ of brain homogenate, the validated lower limit of quantification was $250.0 \mathrm{ng} / \mathrm{mL}$. Intra- and inter-day accuracy and precision were within $15 \%$ of nominal concentrations. Likewise, the analyte and its internal standard proved to be stable during storage, sample preparation and chromatographic analysis.
\end{abstract}

\section{INTRODUCCIÓN}

El potencial de Lepidium meyenii (Maca) se basa en sus innumerables y diversas propiedades farmacológicas. El efecto neuroprotector del extracto pentanico de la Maca ha abierto nuevas vías de investigación para tratar enfermedades del Sistema Nervioso [1]. Las macamidas, metabolitos secundarios de la Maca, serían responsables de dicho efecto, ya que inhiben la actividad de la amida hidrolasa de ácidos grasos (FAAH) [2]. Esta enzima cataboliza la anandamida [3], uno de los principales agonistas de los receptores CB1 del Sistema Endocannabinoide que modula

Correspondencia:

Rita Nieto Montesinos

rnieto@ucsm.edu.pe la liberación de neurotransmisores [2]. Así, las macamidas permitirían cantidades significativas de anandamida en las sinapsis canabinérgicas y una actividad correcta del Sistema Endocannabinoide [3]. Las macamidas obtenidas por síntesis en el laboratorio presentarían los mismos efectos beneficiosos que las macamidas naturales. Es así que a la actualidad, se ha sintetizado 11 de las 19 macamidas naturales [4] y de estas, la macamida sintética N-3-metoxibencil-linoleamida posee comprobado efecto inhibitorio de la FAAH in vitro e in vivo $[4,5]$. En tal sentido, N-3-metoxibencil-linoleamida podría representar una alternativa potencial en el tratamiento de la ansiedad, depresión, dolor crónico, epilepsia, entre otros [5, $6]$.

Con el fin de investigar exhaustivamente la distribución cerebral pre-clínica, es necesario contar con un método analítico que permita cuantificar N-3-metoxibencillinoleamida en dicha matriz. Estudios anteriores han descrito métodos analíticos para la identificación y cuantificación de macamidas en extractos obtenidos a partir de Lepidium 
meyenii por Cromatografía Líquida de Ultra Alta Resolución acoplada a Espectrometría Electrospray de Masas en tándem con Analizador Cuadrupolo (UHPLC-ESI-QTOF-MS/MS) [7] o Cromatografía Líquida de Ultra Alta Resolución acoplada a Espectrometría Electrospray de Masas con Analizador Orbitrap (UHPLC-ESI-OrbitrapMS) [8]. Asimismo, se ha descrito un método analítico para cuantificación de la macamida N-3-metoxibencilpalmitamida en plasma y diversos órganos por Cromatografía Líquida de Ultra Resolución acoplada a un espectrómetro de masas en tándem (UPLC-MS/MS) [9]. Sin embargo, una extensa revisión bibliográfica reveló la falta de métodos analíticos simples y accesibles para la cuantificación de N-3metoxibencil-linoleamida en matriz cerebral. Así, el presente estudio tuvo como objetivo desarrollar un nuevo método analítico por Cromatografía Líquida de Alta Resolución acoplada a un detector de arreglo de diodos para la cuantificación de N-3-metoxibencil-linoleamida en pequeños volúmenes de homogenizado de cerebro de ratas Sprague Dawley. En el presente método se validó la selectividad, límite inferior de cuantificación (LIC), curva de calibración, exactitud, precisión, recuperación y estabilidad según la Guía de Validación de Métodos Bioanalíticos de la Food and Drug Administration (FDA) [10].

\section{MATERIAL Y MÉTODOS}

\section{Material}

Las macamidas sintéticas N-3-metoxi-bencillinoleamida y N-bencilpalmitamida fueron sintetizadas por el Dr. Mark Böhlke del Massachusetts College of Pharmacy and Health Sciences University (Boston, Estados Unidos). Metanol grado HPLC, acetonitrilo grado HPLC y cloruro de sodio fueron adquiridos de Merck (Perú) y el tetraglicol y polietilenglicol 600 (PEG600) de Sigma Aldrich (Estados Unidos). La Heparina de 5000 UI/mL fue adquirida de PharmGen (Perú) y el anestésico Ket-A-Xyl ${ }^{\circledR}$ de Agrovet Market (Perú). El homogenizado de cerebro blanco fue obtenido de ratas Sprague Dawley con un peso entre 250 y 280 gramos.

\section{Preparación de soluciones stock, estándares de calibración (EC) y muestras de control de calidad (MCC)}

Las soluciones stock de N-3-metoxibencil-linoleamida para EC y MCC se prepararon por separado disolviendo la macamida en metanol a una concentración de $1000 \mu \mathrm{g} / \mathrm{mL}$. Las soluciones intermedias fueron preparadas por dilución sucesiva de una solución stock, igualmente en metanol.

Siete EC fueron preparados diluyendo una solución intermedia de N-3-metoxibencil-linoleamida en homogenizado de cerebro para obtener concentraciones de $10000,7500,5000,1000,750,500$ y $250 \mathrm{ng} / \mathrm{mL}$. Tres MCC fueron preparadas diluyendo una solución intermedia de N-3-metoxibencil-linoleamida en homogenizado de cerebro para obtener concentraciones de 7500 (MCC alto), 3000 (MCC medio) y 750 (MCC bajo) ng/mL. La solución stock de estándar interno (SI), N-bencil-palmitamida, fue preparada en metanol a una concentración de $1000 \mu \mathrm{g} / \mathrm{mL}$.

\section{Preparación de muestras}

Previo al análisis cromatográfico, se pesó $500 \mu \mathrm{g}$ de cada muestra de homogenizado de cerebro y se añadió la solución de SI de tal forma que la N-bencil-palmitamida se encuentre a una concentración final de $1000 \mathrm{ng} / \mathrm{mL}$. Luego de la desproteinización por adición de $500 \mu \mathrm{L}$ de acetonitrilo, se agito las muestras por 3 minutos y se centrifugó a 10000 g por 10 minutos. La fase orgánica superior fue decantada y evaporada hasta sequedad y el residuo fue reconstituido con $250 \mu \mathrm{L}$ de metanol. Todos los EC, MCC y muestras fueron procesadas siguiendo el mismo procedimiento.

\section{Instrumentación: Condiciones cromatográficas}

Los análisis cromatográficos fueron realizados en un Cromatográfo Líquido HITACHI equipado con una bomba cuaternaria, un inyector automático, un horno de columna y un detector de arreglo de diodos. El analito se separó en una columna Chromolith ${ }^{\circledR}$ Performance con elución isocrática de acetonitrilo-agua ultrapura $(80: 20, \mathrm{v} / \mathrm{v})$ a $1.0 \mathrm{~mL} / \mathrm{min}$, a $40^{\circ} \mathrm{C}$ y la detección se realizó con el detector de arreglo de diodos a una longitud de onda de $205 \mathrm{~nm}$. El volumen de inyección de cada estándar o muestra fue de $20 \mu \mathrm{L}$.

\section{Procedimiento de validación}

El presente método analítico se validó de acuerdo a la Guía de Validación de Métodos Bio-analíticos de la FDA, 2018 [10]. Así, se evaluó la selectividad, límite inferior de cuantificación (LIC) y curva de calibración, exactitud y precisión intra e inter-día, recuperación absoluta y la estabilidad de 3-metoxibencil-linoleamida.

\section{Selectividad}

La selectividad del método se determinó en seis lotes diferentes de homogenizado de cerebro blanco, los cuales se obtuvieron de seis ratas Sprague Dawley sanas con un peso entre 250 y 280 gramos.

\section{Límite inferior de cuantificación (LIC) y curva de calibración}

El LIC se definió como la concentración del estándar más bajo en la curva de calibración de estándares, donde el pico del analito debe ser reproducible con una exactitud y precisión menor o igual al $20 \%$ de la concentración nominal. Para determinar la linealidad, cada uno de los tres días de validación, se preparó una curva de calibración de siete concentraciones diferentes, 10 000, 7 500, 5 000, 1 000, 750, 500 y $250 \mathrm{ng} / \mathrm{mL}$. Las curvas de calibración se evaluaron mediante regresión lineal del ratio del área de los picos (área de pico de analito / área de pico de SI) (y) frente a la concentración nominal (x) de cada EC. A partir de las curvas individuales correspondientes, se calculó la pendiente, el intercepto y el coeficiente de correlación. El criterio de aceptación para cada concentración estándar fue de $\pm 15 \%$ de la concentración nominal, excepto para la concentración más baja, donde la desviación no debe exceder el $20 \%$. 


\section{Exactitud y precisión}

La exactitud y precisión intra e inter-día se evaluaron mediante la determinación de las concentraciones de N-3metoxibencil-linoleamida en seis repeticiones de cada MCC. La exactitud se calculó como la relación de las concentraciones experimentales y nominales: Exactitud (\%) $=$ (concentración experimental / concentración nominal $) \mathrm{x}$ 100. La precisión se calculó como el coeficiente de variación (CV) de las concentraciones experimentales: CV (\%) = (desviación estándar / promedio) x 100. Los criterios para la aceptación de los datos incluyeron una exactitud dentro del $\pm 15 \%$ de los valores nominales y una precisión dentro del \pm $15 \%$, excepto para el LIC, donde la exactitud y precisión no deben exceder el $20 \%$.

\section{Recuperación absoluta}

La recuperación absoluta de N-3-metoxibencillinoleamida se evaluó comparando la respuesta del analito añadido pre-extracción y post-extracción. Recuperación absoluta $(\%)=$ área del pico del analito añadido pre-extracción / área del pico del analito añadido post-extracción x 100 . La recuperación absoluta de N-3-metoxibencil-linoleamida se evaluó en seis repeticiones de tres concentraciones diferentes de MCC, mientras que la recuperación absoluta del SI se determinó solamente a la concentración utilizada. La recuperación tanto del analito como del SI debería ser consistente y reproducible.

\section{Estabilidad}

La estabilidad de N-3-metoxibencil-linoleamida y su SI en homogenizado de cerebro se evaluaron en seis repeticiones de dos concentraciones (baja y alta) de MCC, expuestas a diferentes temperaturas y tiempos de almacenamiento. Los resultados se compararon con aquellos obtenidos de cada MCC recién preparada. La estabilidad post-congelación y descongelación se evaluó después de tres ciclos de congelación y descongelación. En cada ciclo, las muestras se congelaron a $-20^{\circ} \mathrm{C}$ durante 24 horas y se descongelaron a temperatura ambiente. La estabilidad a corto plazo se determinó después de la incubación de las muestras a temperatura ambiente durante 5 y 24 horas. La estabilidad a largo plazo se evaluó después del almacenamiento de las muestras a $-20^{\circ} \mathrm{C}$ durante 30 y 90 días. La estabilidad postpreparativa se investigó después del almacenamiento a $20^{\circ} \mathrm{C}$ (temperatura del inyector automático) durante 24 horas. La estabilidad de las soluciones stock de 3-metoxibencillinolamida y su SI se evaluó después del almacenamiento a temperatura ambiente durante 6 horas.

\section{RESULTADOS}

\section{Selectividad}

La figura 1 muestra el cromatograma representativo obtenido de homogenizado de cerebro blanco. De acuerdo con el análisis, los componentes de la matriz no interfirieron con los picos de N-3-metoxibencil-linoleamida o N-bencilpalmitamida en sus tiempos de retención, 5.4 y 7.9 minutos, respectivamente, y en el rango de concentración descrito en este manuscrito.

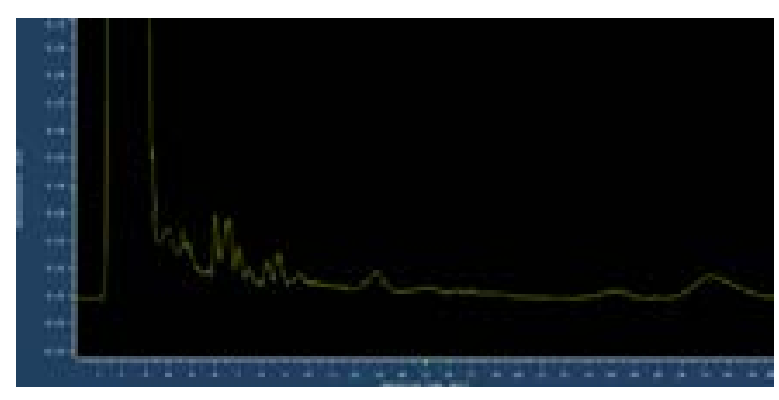

Fig. 1: Cromotagrama representativo de homogenizado de cerebro blanco

Límite inferior de cuantificación (LIC) y curva de calibración

Utilizando $500 \mu \mathrm{g}$ de homogenizado de cerebro de rata, el LIC se definió como la concentración más baja de estándares de las curvas de calibración, $250.0 \mathrm{ng} / \mathrm{mL}$, concentración adecuada para el presente estudio de distribución cerebral. En el LIC, la exactitud inter-día fue de $115.4 \%$ y la precisión inter-día fue de $1.31 \%$ (Tabla 1 ).

En los tres días de validación, se demostró una regresión lineal de la relación del área de los picos frente al rango de concentraciones entre 250 y $10000 \mathrm{ng} / \mathrm{mL}$ (y $=0.0035 \mathrm{x}-$ $0.5430, \mathrm{r} 2>0.9950$ ). La exactitud inter-día de los estándares de calibración osciló entre 93.7 y $112.4 \%$, mientras que la precisión inter-día varió de 0.3 a 7.6\% (Tabla 1).

Tabla 1: Ensayo de linealidad del método de cromatografía líquida de alta resolución para la determinación de N-3metoxibencil-linoleamida en homogenizado de cerebro

\begin{tabular}{cccc}
\hline $\begin{array}{c}\text { Concentración } \\
\text { nominal (ng/g) }\end{array}$ & $\begin{array}{c}\text { Concentración } \\
\text { hallada (ng/g) }\end{array}$ & Exactitud (\%) & Precisión (\%) \\
\hline 250 & 281.0 & 112.4 & 7.6 \\
500 & 486.2 & 97.2 & 3.8 \\
750 & 702.7 & 93.7 & 1.7 \\
1000 & 956.4 & 95.6 & 1.1 \\
5000 & 5000.9 & 100.0 & 0.3 \\
7500 & 7592.3 & 101.2 & 2.4 \\
10000 & 9822.3 & 98.2 & 0.7 \\
\hline
\end{tabular}

\section{Exactitud y precisión}

La exactitud y precisión intra e inter-día para N-3metoxibencil-linoleamida se evaluaron mediante la extracción y el análisis de seis repeticiones de cada uno de las tres MCC en cada uno de los tres días de validación. Las tablas 2 y 3 resumen la exactitud y precisión intra e inter-día para N-3-metoxibencil-linoleamida. 
Tabla 2: Exactitud y precisión intra-día para la cuantificación de N-3-metoxibencil-linoleamida en homogenizado de cerebro

\begin{tabular}{cccc}
\hline Concentración nominal (ng/g) & Día & $\begin{array}{c}\text { Exactitud } \\
(\%)\end{array}$ & $\begin{array}{c}\text { Precisión } \\
(\%)\end{array}$ \\
\hline \multirow{2}{*}{750} & Día 1 & 93.4 & 5.1 \\
& Día 2 & 94.8 & 6.8 \\
& Día 3 & 105.3 & 4.9 \\
& Día 1 & 100.5 & 8.1 \\
\multirow{2}{*}{3000} & Día 2 & 91.2 & 3.0 \\
& Día 3 & 106.4 & 3.5 \\
& Día 1 & 106.5 & 4.9 \\
& Día 2 & 95.6 & 3.2 \\
& Día 3 & 104.4 & 2.3 \\
\hline
\end{tabular}

Tabla 3: Exactitud y precisión inter-día para la cuantificación de N-3-metoxibencil-linoleamida en homogenizado de cerebro

\begin{tabular}{ccc}
\hline $\begin{array}{c}\text { Concentración nominal } \\
(\mathrm{ng} / \mathrm{g})\end{array}$ & Exactitud (\%) & Precisión (\%) \\
\hline 750 & 97.8 & 6.6 \\
3000 & 99.4 & 7.7 \\
7500 & 102.6 & 5.7 \\
\hline
\end{tabular}

\section{Recuperación absoluta}

En el presente estudio, el uso de tert-butil metil éter solo o con acetonitrilo (1:1) o metanol como solventes de extracción dieron lugar a valores de recuperación bajos (estudio piloto). Sin embargo, el uso de acetonitrilo dio lugar a valores de recuperación satisfactorios no solo para 3-metoxibencillinoleamida pero también N-bencil-palmitamida. El valor promedio de recuperación absoluta para N-3-metoxibencillinoleamida es $105.95 \pm 5.2 \%$ y para N-bencil-palmitamida es $89.7 \pm 2.0 \%$.

\section{Estabilidad}

Los resultados de las pruebas de estabilidad, tabla 4, demostraron que el analito de interés se mantuvo estable durante el almacenamiento, la preparación y el análisis cromatográfico de la muestra.

Tabla 4: Estabilidad de N-3-metoxibencil-linoleamida en homogenizado de cerebro

\begin{tabular}{|c|c|c|c|c|c|c|c|}
\hline \multirow{3}{*}{\multicolumn{2}{|c|}{ Prueba de estabilidad }} & \multicolumn{6}{|c|}{ Concentración nominal (ng/g) } \\
\hline & & \multicolumn{2}{|c|}{750} & \multicolumn{2}{|c|}{3000} & \multicolumn{2}{|c|}{7500} \\
\hline & & Exactitud ( & isión (\%) & $\begin{array}{c}\text { Exactitud } \\
(\%)\end{array}$ & $\begin{array}{l}\text { Precisión } \\
(\%)\end{array}$ & $\begin{array}{c}\text { Exactitud } \\
(\%)\end{array}$ & Precisión (\%) \\
\hline \multirow[t]{3}{*}{ Congelación - descongelación } & ler ciclo & 96.7 & 2.1 & 100.4 & 4.8 & 101.7 & 2.1 \\
\hline & 2do ciclo & 95.8 & 2.4 & 99.0 & 4.0 & 102.0 & 3.0 \\
\hline & 3er ciclo & 94.8 & 3.8 & 100.3 & 3.2 & 102.0 & 1.5 \\
\hline \multirow{2}{*}{ En mesa de trabajo } & 5 horas & 100.8 & 0.8 & 100.6 & 1.5 & 100.7 & 2.2 \\
\hline & 24 horas & 94.5 & 5.0 & 99.1 & $2.4 \mathrm{v}$ & 99.4 & 0.9 \\
\hline \multirow{2}{*}{ A largo plazo } & 1 mes & 96.7 & 4.1 & 103.2 & 4.4 & 105.9 & 3.4 \\
\hline & 3 meses & 99.3 & 2.7 & 98.8 & 3.5 & 102.7 & 1.7 \\
\hline Muestra procesada & Inyector automático 24 horas & 95.8 & 3.4 & 99.7 & 2.3 & 98.5 & 2.5 \\
\hline Solución stock & 6 horas & 101.4 & 2.0 & 101.7 & 4.7 & 104.0 & 4.7 \\
\hline
\end{tabular}




\section{DISCUSIÓN}

La administración de cualquier "candidato a ser fármaco" en pacientes, requiere del conocimiento exhaustivo de los beneficios y riesgos a los que podrían estar expuestos los seres humanos. La fase pre-clínica determinará si se debe avanzar hacia la fase clínica o buscar otros candidatos a ser fármacos [11]. Es así, que los programas de desarrollo de fármacos se basan en estudios de farmacocinética y biodistribución en animales de experimentación [12]. La determinación de las concentraciones del futuro fármaco en diferentes tejidos requiere de metodologías analíticas y equipos que proporcionen resultados fiables y que permitan una correcta estimación de los sitios de acción y posibles perfiles tóxicos o reacciones fatales $[12,13]$.

Aunque se ha reportado un método analítico para la cuantificación de la macamida N-3-metoxibencil-palmitamida en cerebro de ratas Sprague Dawley [9], a la actualidad no existen métodos analíticos para la identificación y cuantificación de la macamida N-3-metoxibencil-linoleamida en matrices biológicas. Es así que el presente estudio tuvo como objetivo desarrollar un nuevo método analítico por Cromatografía Líquida de Alta Resolución acoplada a un detector de arreglo de diodos para la cuantificación de N-3metoxibencil-linoleamida en homogenizado de cerebro de ratas Sprague Dawley. Para tal método, se validó la selectividad, límite inferior de cuantificación (LIC), curva de calibración, exactitud, precisión, recuperación y estabilidad según la Guía de Validación de Métodos Bioanalíticos de la Food and Drug Administration (FDA) [10]. La selectividad del método es adecuada ya que no se han encontrado interferencias analíticas provenientes de la misma matriz. La exactitud y precisión hallada para la curva de calibración son adecuadas ya que están dentro de los criterios de aceptación de no más de $20 \%$ de desviación para las concentraciones en el LIC y no más del 15\% de desviación de las concentraciones nominales de los estándares de calibración. De igual manera, la exactitud y precisión de las MCC están dentro de los valores de no más del $15 \%$ de desviación como lo estipula la Guía de Validación de Métodos Bioanalíticos de la Food and Drug Administration (FDA) [10]. El valor promedio de recuperación absoluta para N-3-metoxibencil-linoleamida es $105.95 \pm 5.2 \%$ y para N-bencil-palmitamida es $89.7 \pm 2.0 \%$. Este último valor se aproxima a los valores obtenidos $(80.3$ $-86.2 \%$ ) en un estudio anterior el cual tuvo como objetivo cuantificar N-3-metoxibencil-palmitamida en cerebro de ratas Sprague Dawley por UPLC-MS/MS [9]. Sin embargo, tal estudio utilizó ácido fórmico y etanol además de una columna de extracción en fase sólida para la extracción de macamidas, mientras que la extracción en el presente estudio fue sencilla ya que solo se utilizó acetonitrilo. Asimismo, la macamida sintética N-3-metoxibencil-linoleamida demostró ser estable bajo diferentes condiciones, lo cual posibilita que el método analítico propuesto en este manuscrito pueda ser aplicado a estudios de distribución cerebral y luego de una semi-validación a estudios de bio-distribución y farmacocinética en modelos murinos.

En conclusión, la simplicidad de este método permitirá identificar y cuantificar de manera práctica los niveles de la macamida sintética N-3-metoxibencil-linoleamida en cerebro de animales murinos mediante HPLC.

\section{AGRADECIMIENTOS}

Al Fondo Nacional de Desarrollo Científico, Tecnológico y de Innovación Tecnológica (FONDECYT), por el financiamiento del presente proyecto a través del contrato 096 - 2015 - FONDECYT. Al Vicerrectorado de Investigación de la Universidad Católica de Santa María por el apoyo económico y logístico.

\section{REFERENCIAS BIBLIOGRÁFICAS}

1. Pino-Figueroa, A., D. Nguyen, and T.J. Maher, Neuroprotective effects of Lepidium meyenii (Maca). Ann N Y Acad Sci, 2010. 1199: p. 77-85.

2. Alasmari, M., et al., Inhibition of Fatty Acid Amide Hydrolase (FAAH) by Macamides. Mol Neurobiol, 2019. 56(3): p. 1770-1781.

3. Huyen Vu, A.P.F., Timothy Maher, Fatty Acid Amide Hydrolase (FAAH) Inhibitors: Discovery in Lepidium meyenii (Maca) Extracts. Proceedings of The National Conference On Undergraduate Research (NCUR) 2012, 2012.

4. Wu, H., et al., Macamides and their synthetic analogs: evaluation of in vitro FAAH inhibition. Bioorg Med Chem, 2013. 21(17): p. 5188-97.

5. Almukadi, H., et al., The macamide N-3methoxybenzyl-linoleamide is a time-dependent fatty acid amide hydrolase (FAAH) inhibitor. Mol Neurobiol, 2013. 48(2): p. 333-9.

6. Romigi, A., et al., Cerebrospinal fluid levels of the endocannabinoid anandamide are reduced in patients with untreated newly diagnosed temporal lobe epilepsy. Epilepsia, 2010. 51(5): p. 768-72.

7. Chen, S.X., et al., Optimization of Ultrasound-Assisted Extraction, HPLC and UHPLC-ESI-Q-TOF-MS/MS Analysis of Main Macamides and Macaenes from Maca (Cultivars of Lepidium meyenii Walp). Molecules, 2017. 22(12).

8. Zhou, Y., et al., Chemical profiling analysis of Maca using UHPLC-ESI-Orbitrap MS coupled with UHPLCESI-QqQ MS and the neuroprotective study on its active ingredients. Sci Rep, 2017. 7: p. 44660.

9. Qihong Zhang, K.W., Yong Xu, Saidan Ding, Faqin Ye and Xuebao Wang, Pharmacokinetics and tissue distribution of N-3-methoxybenzyl-palmitamide in rat: A macamide derived from Lepidiummeyenii. Tropical Journal of Pharmaceutical Research 2017. 16(8): p. 2039 - 2046.

10. U.S. Departement of Health and Human Services, F.a.D.A., Guidance for Industry: Bioanalytical Method Validation. 2001.

11. Petrova, E., Innovation and Marketing in the Pharmaceutical Industry. Springer Science+Business Media New York 2014, 2014. Innovation in the Pharmaceutical Industry: The Process of Drug Discovery and Development.

12. Ying Huang, M.H., Denise Gavin, Mercedes Serabian, Lee Lee Ong, Maritza C McIntyre, Nicolas Ferry, Akihiro Kume, Dino Petrin, Ying-Hsien Fu, Andreas Marti, Fabricio Oliveira, Sumol Pavittranon and Won Shin, Biodistribution studies: understanding 
international expectations. Molecular Therapy Methods \& Clinical Development, 2016. 3(16022).

13. Hughes, J.P., et al., Principles of early drug discovery. Br J Pharmacol, 2011. 162(6): p. 1239-49. 